\title{
Horizontal Well Productivity Evaluation For Stress Sensitive Elliptical Reservoirs
}

\author{
Weidong Tian, Zhenzhen Dong* and Jiaen Lin, Xi'an Shiyou University, Xi'an, China
}

\begin{abstract}
Well productivity model is one of the vital tools required to evaluate well performance. Most horizontal well productivity models are idealistic in nature, mainly developed for homogeneous reservoirs and conventional reservoirs, and ignore the influences of the pore pressure and stress changes. However, as the capillary in low permeability porous media is tiny, the medium permeability is quite sensitive to pressure change. Thus, there is an urgent need for new realistic productivity models that describe the actual reservoir inflow performance behavior more efficiently than the available models.

This paper presents a new horizontal well productivity model which accounts for the stress sensitive permeability in an elliptical reservoir. Then, the proposed model was extended to investigate the effects of reservoir heterogeneity, eccentricity, and formation damage on horizontal well productivity. The results show that the thinner the formation is, the greater the impact of the horizontal well lengths on production. As the horizontal well length is longer, the impact of stress sensitivity on the production becomes more significant. Horizontal well would be a better well type option for elliptical reservoirs. The longer the horizontal well is, the more impact of heterogeneity, eccentricity distance, as well as skin factor on productivity.

The new model provides a simpler and more reliable means to optimize horizontal well length and efficiently forecast well behavior in stress sensitive reservoirs, such as tight gas reservoir and shale oil reservoirs, with respect to horizontal well productivity to vertical well productivity.
\end{abstract}

\section{Introduction}

To determine the economic feasibility of drilling a horizontal well, the engineers need reliable methods to estimate its expected productivity. There have been attempts to describe and estimate horizontal well productivity.

Joshi (1988a) further illustrates the principle of horizontal well production through electrical simulation, and the calculation of steady-state production of horizontal well was derived in detail. Up to now, most of the steady-state horizontal well productivity formulas proposed by many authors are similar to the formulas. Larsen (1996) proposed a method for calculating the productivity equation of multilateral wells, branch wells and other generalized wells.

Babu and Odeh (1989) calculated the productivity equation of the horizontal well in a pseudo-steady state. Billiter et al. (2001) proposed the dimensionless inflow dynamic curve of the non-fracture horizontal gas well. The flow equation of the Babu and Odeh's horizontal well is transformed into the pseudo-pressure form of the gas well, and the non-darcy flow effect as well as the mechanical skin effect are considered. Furthermore, the pseudo-steady horizontal well productivity of anisotropic reservoir (Lu and Tiab 2007),

Copyright $($ ) the author(s). This work is licensed under a Creative Commons Attribution 4.0 International License. 
constant rate and constant pressure (Hagoort 2011) are given. Salam (2019) presents a new practical method for determining the start time of pseudo-steady flow and constant-behavior productivity index (PI).

Other scholars have considered the wellbore flow into the productivity formula. Penmatcha and Khalid (1999) proposed a semi-analytical model for homogeneous reservoirs that can quantify the impact of wellbore single-phase and two-phase oil and gas in wellbores on productivity. Anklam and Wiggins (2005) presented a model for estimating horizontal well productivity, which combined wellbore fluid dynamics to calculate the well pressure for the entire wellbore. Zhu et al. (2002) (for multilateral wells) and Yildiz (2003) (for perforated horizontal wells) have done similar research. Guo, et al. (2006) developed a general mechanistic model combining the fluid flow of a single branch. The model strictly considers the pressure drop in the vertical and inclined wellbore sections.

In recent years, scholars have been mainly devoted to the study of the productivity of horizontal wells with tight reservoirs, taking into account the factors such as multi-layer reservoir, capillary force, hydraulic fracturing and so on. Tabatabaei et al. (2009) in studying the yield of horizontal wells for hydraulic fracturing in Bakken shale reservoir, established an analytical model to predict the horizontal well yield for longitudinal fractures in multi-layer reservoirs. Kewen and Chen (2012) derived formulas for calculating water cut and dimensionless total and oil productivity indices (PIs) by considering capillary pressure, to study the effect of capillary pressure on production performance in low-permeability oil wells or reservoirs. Bin et al. (2015) present a new analytical solution to study the interplay between flowing pressure and production rate for horizontal well completed within stimulated reservoir volumes (SRV) in tight gas reservoirs. Chen et al. (2019) presented the calculation method of fracturing production of horizontal well through layer, considering the inter-slit interference and wellbore interference. Sun et al. (2019) set up a seepage model for the tiny reservoir by coupling the elliptical flow in the matrix and the near radial flow in the fracture.

All of the above pseudo-steady state equations are either too complicated to use or very time consuming. Moreover, they all ignored the influences of the pore pressure and stress changes on horizontal wells. However, as the capillary in low permeability porous media is tiny, the medium permeability is quite sensitive to pressure change. The effect of pressure on permeability cannot be ignored, especially for the abnormally high pressure and low permeability reservoirs. This paper provides analytical equations to calculate productivity of horizontal wells in low-permeability reservoirs with considering the effect of stress on permeability. Then, the effect of shape of drainage area, heterogeneity, eccentricity and formation damage on the proposed horizontal well productivity model were studied. The work discussed here was carried out at Xi'an Shiyou University, from June to December 2019.

\section{Materials and Methods}

\section{Physical model}

Figure 1 is a schematic of a horizontal well. The following assumptions are made:

1. The horizontal well is in the middle of an elliptical reservoir.

2. The horizontal well is in the middle of the formation, with an impermeable top and bottom boundary.

3. The reservoir is homogeneous and isotropic.

4. The length of horizontal well is $L$ and the width of reservoir is $h$.

5. The flow of fluid is slight compressible single-phase flow of oil which corresponds to the low-speed non-Darcy flow law.

6. Ignore the effect of gravity and capillary forces. 


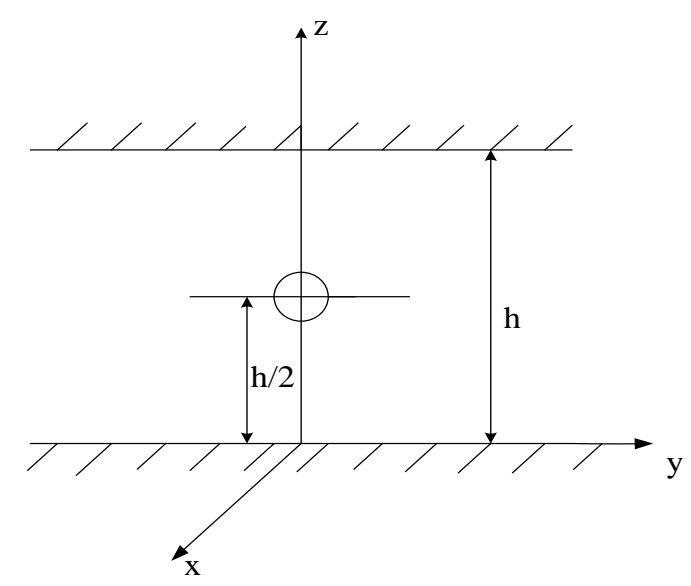

Figure 1-The horizontal well scheme of low permeability reservoir

\section{Productivity Formula Derivation}

The horizontal flow calculations. The horizontal flow of well keeps its shape in ellipses, introducing the $R u$ koves $K i$ function

$$
\frac{z}{L / 2}=\frac{1}{2}\left(\omega+\frac{1}{\omega}\right)
$$

Utilize conformal mapping and transfer the area of elliptical shape with semi-major axis of $a$ as well as semi-minor axis of $b$ into the circular area with radius of $\frac{a+b}{L / 2}$. The segment from $(-\mathrm{L} / 2,0)$ to $(+\mathrm{L} / 2,0)$ is imaged into the unit circle, as shown in Figure 2. The flow on $\omega$ surface can be considered as the supply which is provided from the circular with radius of $\frac{a+b}{L / 2}$ to a vertical well with radius of 1 .
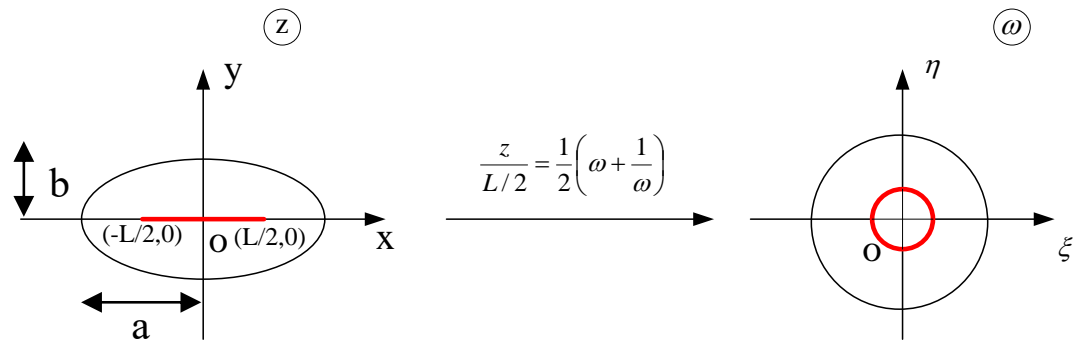

Figure 2-Scheme of horizontal conformal mapping

Most horizontal well productivity models ignored the influences of the pore pressure and stress changes. However, as the capillary in low permeability porous media is tiny, the medium permeability is quite sensitive to pressure change. The effect of pressure on permeability cannot be ignored, especially for the abnormally high pressure and low permeability reservoirs. Many research efforts have shown that the permeability changes exponentially with the pressure. Thus,

$$
K_{D}=\frac{k}{k_{h}}=e^{-\alpha_{k}\left(p_{i}-p\right)}
$$

Consider the existence of starting pressure gradient, so the fluid velocity can be defined as

$v=\frac{k}{\mu}\left[\frac{d p}{d r}-G_{p}\right]$

Substitute Eq. 2 into Eq. 3 and replace the fluid velocity with production to yield 


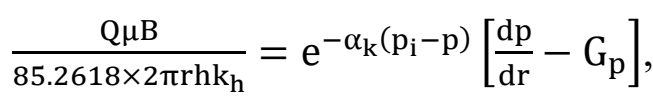

Based on the research of Chen et al. $(2006,2007)$, the oil production formula is as follows.

$$
\mathrm{Q}=\frac{k_{h} h}{1.8665 \times 10^{-3} \alpha_{k} \mu B} \frac{1-e^{\left\{-\alpha_{k}\left[p_{i}-p_{w}-G_{p}\left(r_{e}-r_{w}\right)\right]\right\}}}{\ln \frac{r_{e}}{r_{w}}} .
$$

Consider the property of elliptical $\mathrm{b}=\sqrt{a^{2}-(L / 2)^{2}}$, the horizontal flow of the production wells can be expressed as

$$
\mathrm{Q}_{H}=\frac{k_{h} h}{1.8665 \times 10^{-3} \alpha_{k} \mu B} \frac{1-e^{\left\{-\alpha_{k}\left[p_{i}-p_{W}-G_{p}\left(\frac{a+\sqrt{a^{2}-\frac{L^{2}}{4}}}{L / 2}-1\right)\right]\right\}}}{\ln \left(\frac{a+\sqrt{a^{2}-\frac{L^{2}}{4}}}{L / 2}\right)}, \ldots
$$

where

$$
a=\frac{L}{2} \sqrt{\frac{1}{2}+\sqrt{\frac{1}{4}+\left(\frac{2 r_{e H}}{L}\right)^{4}}}, r_{e H}=\sqrt{A / \pi} \ldots
$$

The vertical flow calculations. The vertical flow of horizontal well can be regarded to be a junction of supply from the top and bottom boundaries. The diagram of vertical conformal transformation is shown in Figure 3.

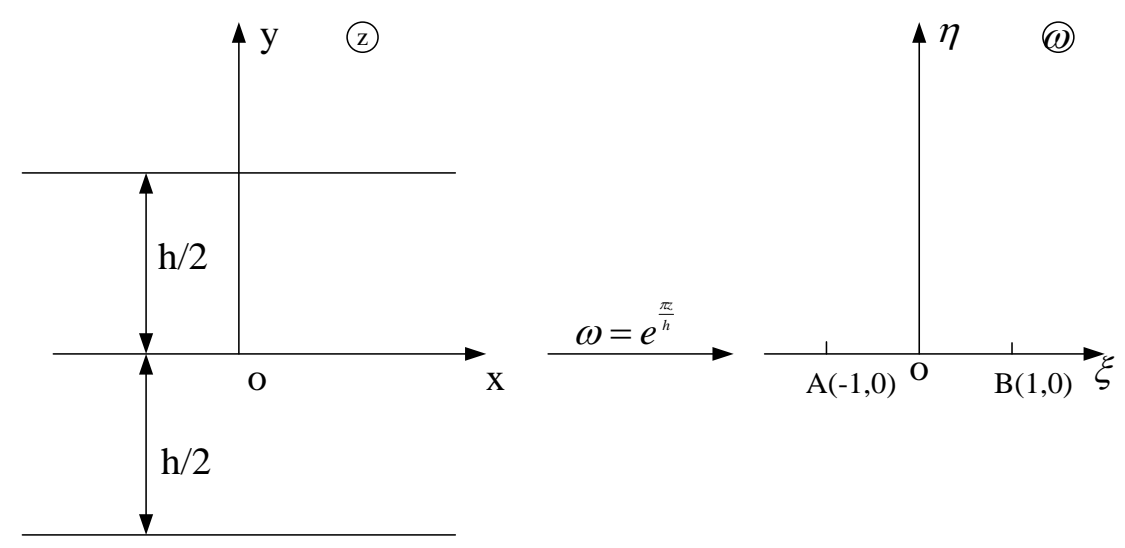

Figure 3-Vertical conformal transformation

Convert the band-shaped region $(-\mathrm{h} / 2<\mathrm{y}<\mathrm{h} / 2)$ on $z$ plane to the unit circle domain on $\omega$ plane. Then, the junction of $(0,0)$ on $z$ plane will be converted to the B point $(1,0)$ on $\omega$ plane and the well radius of $r_{w}$ on $z$ plane will be converted to $\rho_{w}$ on $\omega$ plane.

On $\omega$ plane, the complex potential of any points can be obtained through mirror image theory. Thus,

$$
\mathrm{U}=\frac{q}{2 \pi} \ln (\omega-1)(\omega+1)+C=\frac{q}{4 \pi} \ln \left[\left(\varsigma^{2}+\eta^{2}+1\right)^{2}-4 \varsigma^{2}\right]+i \frac{q}{2 \pi}\left(\operatorname{arctg} \frac{\eta}{\varsigma-1}+\operatorname{arctg} \frac{\eta}{\varsigma+1}\right)+c_{2},
$$

Where $\varsigma^{2}=e^{\frac{2 \pi x}{h}} \cos ^{2} \frac{\pi y}{h} ; \eta^{2}=e^{\frac{2 \pi x}{h}} \sin ^{2} \frac{\pi y}{h}$.

Therefore, the potential function (pressure function) is given as,

$$
\Phi=\frac{q}{4 \pi} \ln \left[\left(e^{\frac{2 \pi x}{h}}+1\right)^{2}-4 e^{\frac{2 \pi x}{h}} \cos ^{2} \frac{\pi y}{h}\right]+c_{2}
$$


When $x=0, \mathrm{y}=\mathrm{h} / 2$, then $\phi_{\frac{h}{2}}=\frac{q}{4 \pi} \ln 4+c_{2}$.

The potential of the junction $B$ on the wall of well can be defined as $\Phi_{w}$ and it is always divided into two parts, which is brought by the junction $A$ and junction $B$, respectively. The distance from junction $A$ to junction $B$ can be approximately equal to 2 and the radius of junction $B$ is $\rho_{w}$. Thus,

$$
\begin{aligned}
& \rho_{w}=\left.\frac{d(\omega-1)(\omega+1)}{d z}\right|_{(0,0)} r_{w}=\left.\frac{2 \pi}{d z} e^{\frac{\pi z}{h}}\right|_{(0,0)} r_{w}=\frac{2 \pi r_{w}}{h}, \\
& \Phi_{w}=\frac{q}{2 \pi}\left[\ln 2+\ln \frac{2 \pi r_{w}}{h}\right]+c_{2}
\end{aligned}
$$

The Non-Darcy flow equation can be expressed as follows

$$
\frac{d \Phi}{d r}=\frac{k}{\mu}\left(\frac{d p}{d r}-G_{p}\right)
$$

The flow on $\omega$ plane can be regarded as the unitary circular supply for a vertical well in one area with radius of $\rho_{w}$ (which will be corrected to the effective caliper using Peaceman method (1990)) whose discharge area is a rectangle with $h / 2$ as long side and $h / 2 \pi$ as short side. The area of this rectangle is equivalent to the area of the circle with a radius of $h / 2 \pi$. Do integration to the Eq.11 within $\left(r_{w}, h / 2\right)$ and according to the Dupuit formula, the shunt volume on the vertical direction of horizontal wells is

$$
\mathrm{Q}_{v}=\frac{k_{h} L}{1.8665 \times 10^{-3} \alpha_{k} \mu B} \frac{1-e^{\left\{-\alpha_{k}\left[p_{e}-p_{w}-G\left(1-\ln \frac{2 \pi r_{w}}{h}\right)\right]\right\}}}{\ln \left(\frac{h}{2 \pi\left(r_{w}+1\right)}\right)} .
$$

Horizontal well productivity. The oil flow resistance on horizontal direction $\left(\Omega_{H}\right)$ and on vertical direction $\left(\Omega_{V}\right)$ which are based on Eq. 6 and Eq.13 can be expressed as follows

$$
\begin{gathered}
\Omega_{H}=\frac{1.8665 \times 10^{-3} \alpha_{k} \mu B}{k_{h} h} \ln \left(\frac{a+\sqrt{a^{2}-\frac{L^{2}}{4}}}{L / 2}\right) . \\
\Omega_{v}=\frac{1.8665 \times 10^{-3} \alpha_{k} \mu B}{k_{h} L} \ln \left(\frac{h}{2 \pi\left(r_{w}+1\right)}\right) \ldots . .
\end{gathered}
$$

Total production of the horizontal well resulting from these two flow components is obtained by adding the respective flow resistances,

$\frac{\Delta \mathrm{p}}{\mathrm{Q}}=\left(\frac{\Delta \mathrm{p}}{\mathrm{Q}_{H}}\right)+\left(\frac{\Delta \mathrm{p}}{\mathrm{Q}_{v}}\right)$,

or pressure drawdown per unit volumetric flow rate,

$\mathrm{Q}=\left(p_{e}-p_{w}\right) /\left(\Omega_{H}-\Omega_{v}\right)$,

Thus, the horizontal well flow rate can be given as,

$$
\mathrm{Q}=\frac{k_{h} h}{1.8665 \times 10^{-3} \alpha_{k} \mu B} \frac{\left.\left.1-e^{\left\{-\alpha_{k}\left[p_{e}-p_{w}-G\left(\frac{a+\sqrt{a^{2}-\frac{L^{2}}{4}}}{L / 2}-\ln \frac{2 \pi r_{w}}{h}\right)\right.\right.}\right]\right\}}{\ln \left(\frac{a+\sqrt{a^{2}-\frac{L^{2}}{4}}}{L / 2}\right)+\frac{h}{L} \ln \left(\frac{h}{2 \pi\left(r_{w}+1\right)}\right)} .
$$

\section{Expansion of the Proposed Productivity Model}

The proposed productivity model (Eq. 18) was extended to consider more practical conditions, such as heterogeneous reservoir, off-center wells, and formation damage.

Heterogeneity. With considering reservoir heterogeneity, the steady flow percolation equation is given as,

$$
k_{x} \frac{\partial^{2} p}{\partial x^{2}}+k_{y} \frac{\partial^{2} p}{\partial y^{2}}+k_{z} \frac{\partial^{2} p}{\partial z^{2}}=0
$$


According to the assumption of Joshi (1988b), the horizontal wells flow in the three-dimensional system is always divided into the horizontal flow and the vertical flow. Therefore, the stable seepage can be simplified as,

$\left(\frac{\partial^{2} p}{\partial x^{2}}\right)+\frac{k_{v}}{k_{h}}\left(\frac{\partial^{2} p}{\partial z^{2}}\right)=0$

Introduce $\mathrm{z}=\mathrm{z} \sqrt{\frac{k_{h}}{k_{v}}}$, then the heterogeneous problem of Eq. 20 can be transformed into a homogeneous problem, expressed in Eq. 19.

$\left(\frac{\partial^{2} p}{\partial x^{2}}\right)+\left(\frac{\partial^{2} p}{\partial z^{2}}\right)=0$

Substitute $h \sqrt{\frac{k_{h}}{k_{v}}}$ for $h$ in Eq.20 and Eq.21, and substitute the average permeability $\sqrt{k_{h} k_{v}}$ or $k_{i}$ in Eq.19, then the horizontal well production formula with considering heterogeneity can be expressed as,

$$
\mathrm{Q}=\frac{k_{h} h}{1.8665 \times 10^{-3} \alpha_{k} \mu B} \frac{\left.\left.1-e^{\left\{-\alpha_{k}\left[p_{e}-p_{w}-G\left(\frac{a+\sqrt{a^{2}-\frac{L^{2}}{4}}}{L / 2}-\ln \frac{2 \pi r_{w}}{h}\right)\right.\right.}\right]\right\}}{\ln \left(\frac{a+\sqrt{a^{2}-\frac{L^{2}}{4}}}{L / 2}\right)+I_{\operatorname{ani} i} \frac{h}{L} \ln \left(\frac{h I_{a n i}}{2 \pi\left(r_{w}+1\right)}\right)},
$$

Where $I_{a n i}=\sqrt{\frac{k_{h}}{k_{v}}}$.

Eccentricity. The eccentricity only affects the vertical plane of horizontal well productivity. Introducing transformation $\omega=\mathrm{z}+\mathrm{i} \delta$ will transform the problem solving with eccentricity into the problem solving without eccentricity, seen in Figure 4.

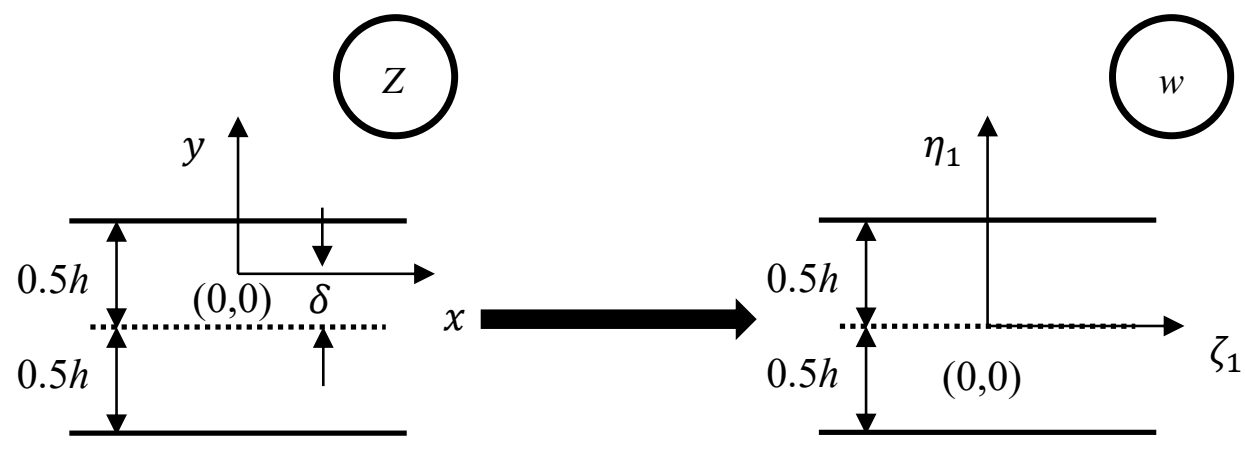

Figure 4-Vertical Conformal Transformation Affected by Eccentricity

Introduce conformal transformation,

$$
\omega=\frac{1-e^{\frac{\pi}{h}(\mathrm{z}+\mathrm{i} \delta)}}{1+e^{\frac{\pi}{h}(\mathrm{z}+\mathrm{i} \delta)}}, \cdots
$$

and convert the band-shaped region with eccentricity on $z$ plane to the unit circle domain on $\omega$ plane. Then, the junction of $(0,0)$ on $z$ plane will be converted to the original point $(0,0)$ on $\omega$ plane and the production well radius of $r_{\mathrm{w}}$ on z plane will be converted to $\rho_{w}$ on $\omega$ plane. Thus,

$$
\rho_{w}=\left.\frac{d \omega}{d z}\right|_{(0,0)} r_{w}=\frac{2 \pi r_{w}}{h\left(2+e^{i \frac{\pi \delta}{h}}+e^{-i \frac{\pi \delta}{h}}\right)}=\frac{\pi r_{w}}{2 h \cos ^{2} \frac{\pi \delta}{2 h}}
$$

According to the study of Yuan et al. (2009 and 2015) and Chen et al.(2009, 2012), for the reservoirs with anisotropic permeability, there is 


$$
J_{h}=\frac{0.543 k_{h} h /\left(\mu_{o} B_{o}\right)}{\ln \left(\frac{a+\sqrt{a^{2}-\frac{L^{2}}{4}}}{L / 2}\right)+\left(\frac{\beta h}{L}\right) \ln \left(\frac{\left(\frac{\beta h}{2}\right)^{2}+\beta^{2} \delta^{2}}{\frac{\beta h}{2} r_{w}}\right)}
$$

Therefore, horizontal well production formula considering the existence of eccentricity can be expressed as,

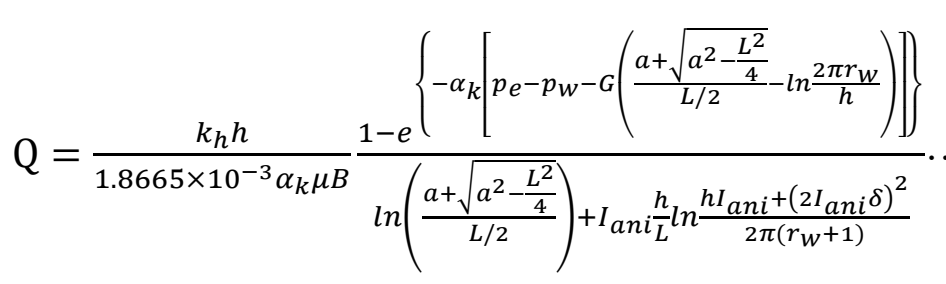

Formation damage. Compared with vertical wells, the drilling time of horizontal wells in the oil layer is much longer, so the contact area between producing formation and drilling fluid is larger. Therefore, under the same drilling conditions, the contamination of horizontal well reservoir is much severer than that of the vertical wells reservoir, resulting in the production difference between horizontal wells and vertical wells. The parameter which is used to represent the horizontal wellbore damage is usually called skin factor, abbreviated as $S$. It is be obtained through drill-stem testing (DST) or pressure recovery testing. The value of $S$ can affect the flow efficiency of the fluid, but it is not the unique factor and it does not exist independently. The flow efficiency of the fluid also can be affected by the vertical penetration level (Giger et al. 1984). Therefore, when the effect of formation damage on horizontal well productivity was studied, the effect of skin and permeability anisotropy should be considered. According to the study of Renard and Dupuy (1991), the value of skin can be estimate roughly based on the following equation,

$$
S_{h}=\frac{I_{\text {ani }} h S}{L} \text {. }
$$

Thus, horizontal well production formula considering the existence of formation damage can be expressed as follows.

$$
\mathrm{Q}=\frac{k_{h} h}{1.8665 \times 10^{-3} \alpha_{k} \mu B} \frac{\left.\left.1-e^{\left\{-\alpha_{k}\left[p_{e}-p_{W}-G\left(\frac{a+\sqrt{a^{2}-\frac{L^{2}}{4}}}{L / 2}-\ln \frac{2 \pi r_{w}}{h}\right)\right.\right.}\right)\right\}}{\ln \left(\frac{a+\sqrt{a^{2}-\frac{L^{2}}{4}}}{L / 2}\right)+I_{a n i} \frac{h}{L}\left(\ln \frac{h I_{a n i}+(2 I a n i}{2 \pi\left(r_{w}+1\right)}+s\right)}
$$

\section{Results and Discussion}

In this section, the proposed production model (Eq. 28) was applied to study the impact of horizontal well length $(L)$, reservoir thickness $(h)$, medium deformation coefficient $\left(a_{k}\right)$, shape of drainage area $(a / b)$, anisotropy index $\left(I_{\text {ani }}\right)$, eccentric distance $(\delta)$ and formation damage $(S)$ on horizontal well production $(Q)$. The basic data that used to calculate production rate are listed in Table $\mathbf{1 .}$ 
Table 1-Basic data used in the calculation

\begin{tabular}{|c|c|c|c|}
\hline Parameters & Value & Parameters & Value \\
\hline$k_{h}, \mu m^{2}$ & 0.1 & $p_{w}, M P a$ & 8 \\
\hline$\alpha_{k}, M P a^{-1}$ & 0.1 & $G, M P a / m$ & 0.001 \\
\hline$\mu, m P a . s$ & 0.9 & $r_{w}, m$ & 0.1 \\
\hline$B$ & 1.2 & $A, m^{2}$ & 300000 \\
\hline$p_{e}, M P a$ & 10 & $h, m$ & 0 \\
\hline$I_{\text {ani }}$ & 1 & $\delta, m$ & \\
\hline$S$ & 0 & & 40 \\
\hline
\end{tabular}

Horizontal well length and reservoir thickness. Figure 5 shows that the production increases as the reservoir thickness and horizontal well length increase. The thinner the formation is, the greater the impact of the horizontal well lengths on production. For instance, as the reservoir thickness increases from $5 \mathrm{~m}$ to $50 \mathrm{~m}$, the horizontal well production increases from 1280.7 to $9941.510^{5} \mathrm{~m}^{3} / \mathrm{d}(7.8$ times) when horizontal well length is set to be $1000 \mathrm{~m}$. While the well length is $3000 \mathrm{~m}$, the production only increases by 5.5 times. Thus, horizontal well is suggested in a thin formation.

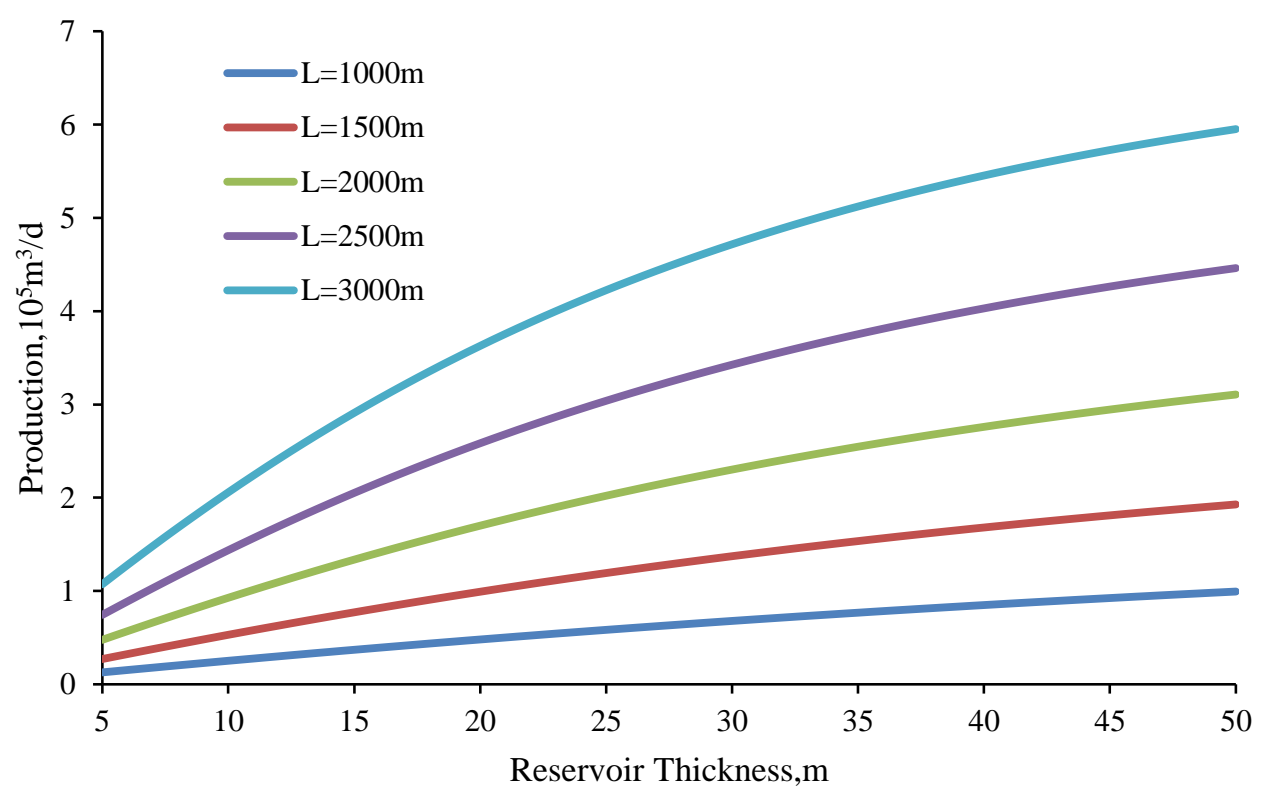

Figure 5-Effect of reservoir thickness on production at different horizontal well length

Medium deformation coefficient. Medium deformation coefficient, $\alpha_{k}$, is an indicator of the degree of stress sensitivity (Eq. 2). Its influence on productivity is shown in Figure 6, which indicates that medium deformation coefficient has a negative linear correlation with production on the semi-log plot. The larger the coefficient, the smaller the production. In other words, the more stress sensitive the formation is, the lower the production. As the horizontal well length is longer, the impact of medium deformation coefficient on the production becomes more significant. 


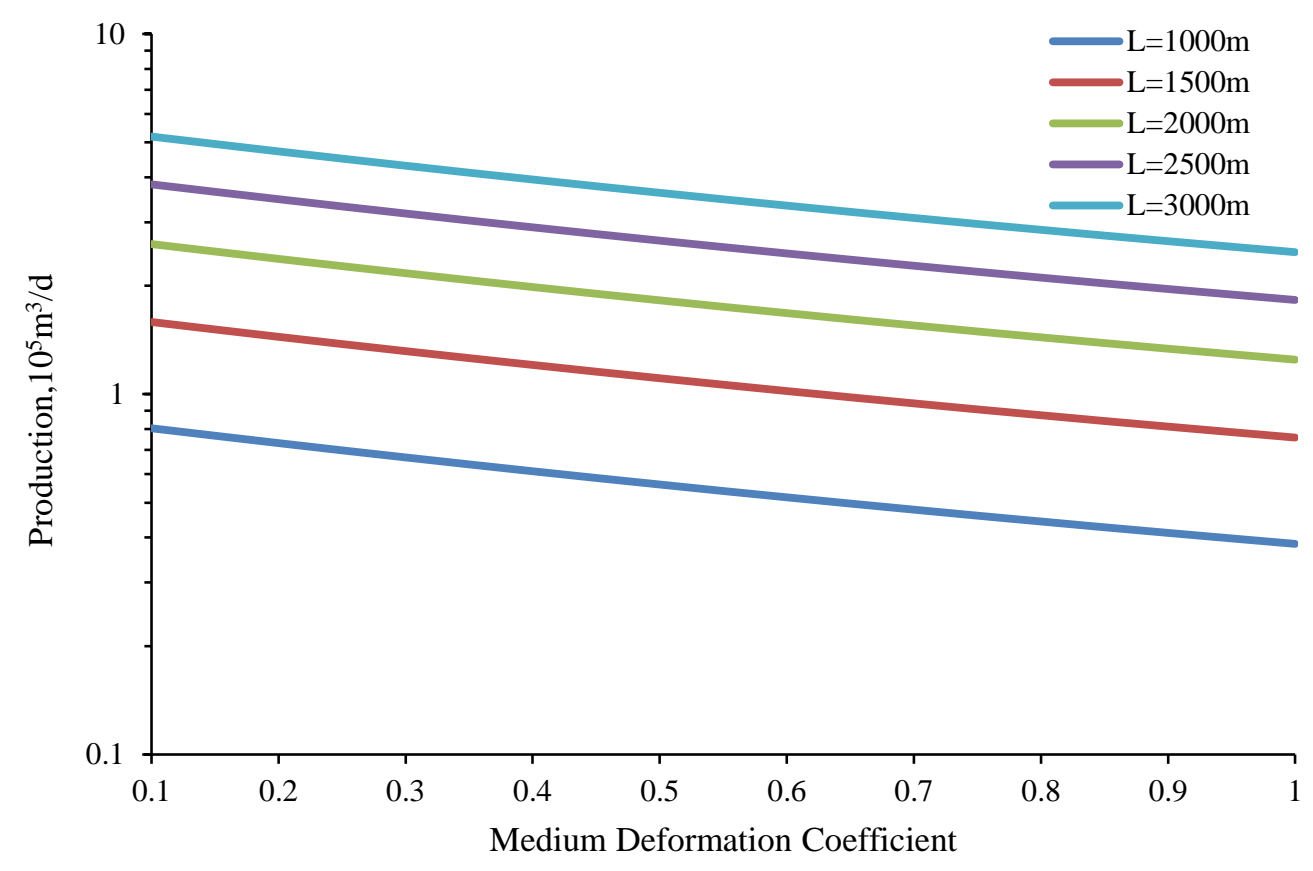

Figure 6-Effect of the medium deformation coefficient on production at different horizontal well length

Shape of drainage area. The shape of drainage area is determined by the ratio $\mathrm{a} / \mathrm{b}$ in Figure 2 . The influence of the medium deformation coefficient on production with various ratio of $\mathrm{a} / \mathrm{b}$ is shown in Figure. 7. The horizontal well length $L$ was set to be $1000 \mathrm{~m}$, other parameters are listed in Table 1. Fig 6 shows that the smaller $a / b$ (the closer reservoir shape is to the circle), the lower the production of horizontal well. Thus, horizontal well would be a better well type for elliptical reservoirs.

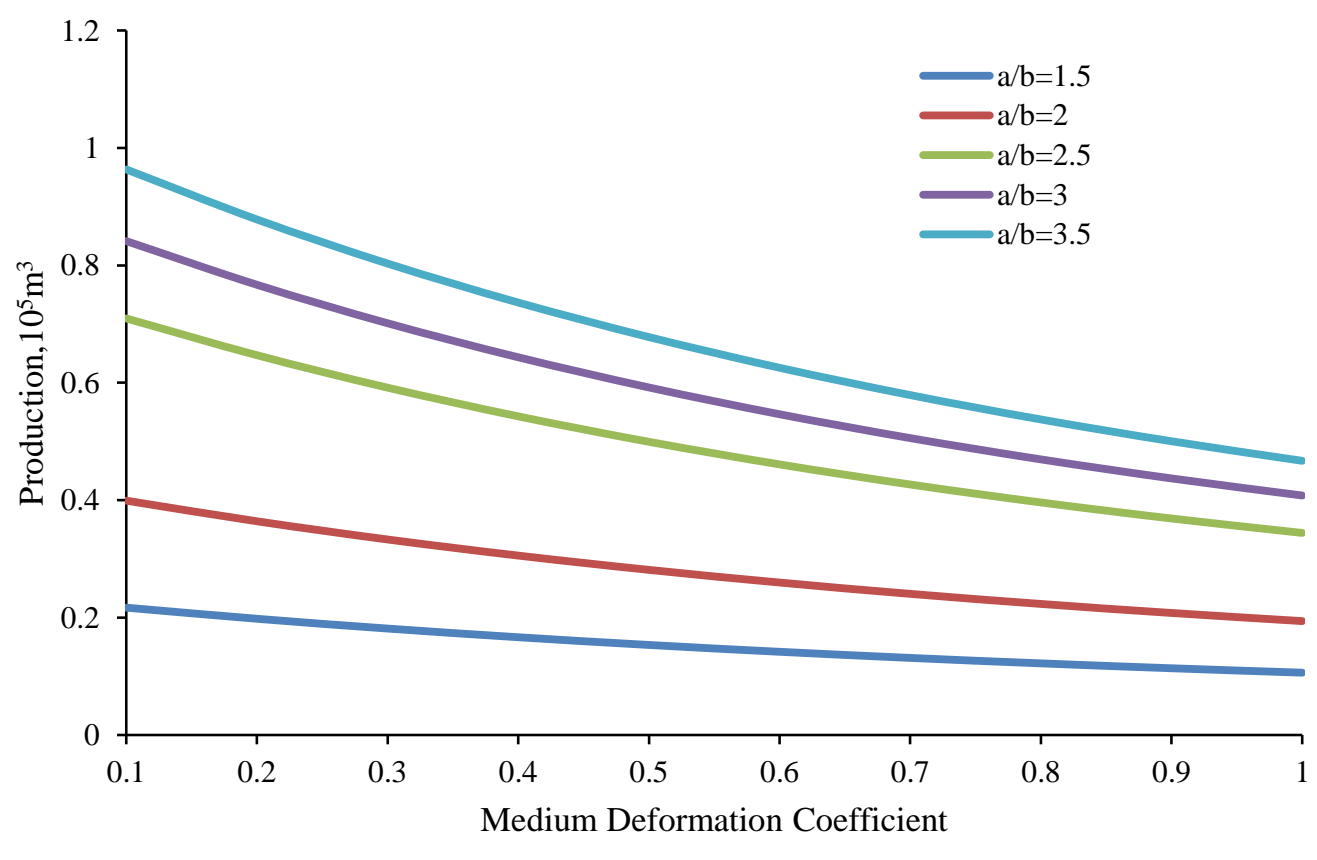

Figure 7-Effect of different $a / b$ on production

Anisotropy index. Anisotropy index is an indicator is an indicator of the degree of reservoir heterogeneity (Eq. 22). The influence of the permeability anisotropy index on production is shown in Figure 8 in terms of different horizontal well length. It shows heterogeneity has great impact on production. The smaller the vertical permeability (the greater the anisotropy index), the lower the horizontal well production. As 
anisotropy index increases from 1 to 10 , the production decreases from 41,024 to $6,01910^{5} \mathrm{~m}^{3} / \mathrm{d}(6.8$ times) when the horizontal well length is $3000 \mathrm{~m}$. While the horizontal well length is $1000 \mathrm{~m}$, the production only decreases 2.6 times. Thus, it can be concluded that the stronger the heterogeneity, the more impact of horizontal well length on productivity.

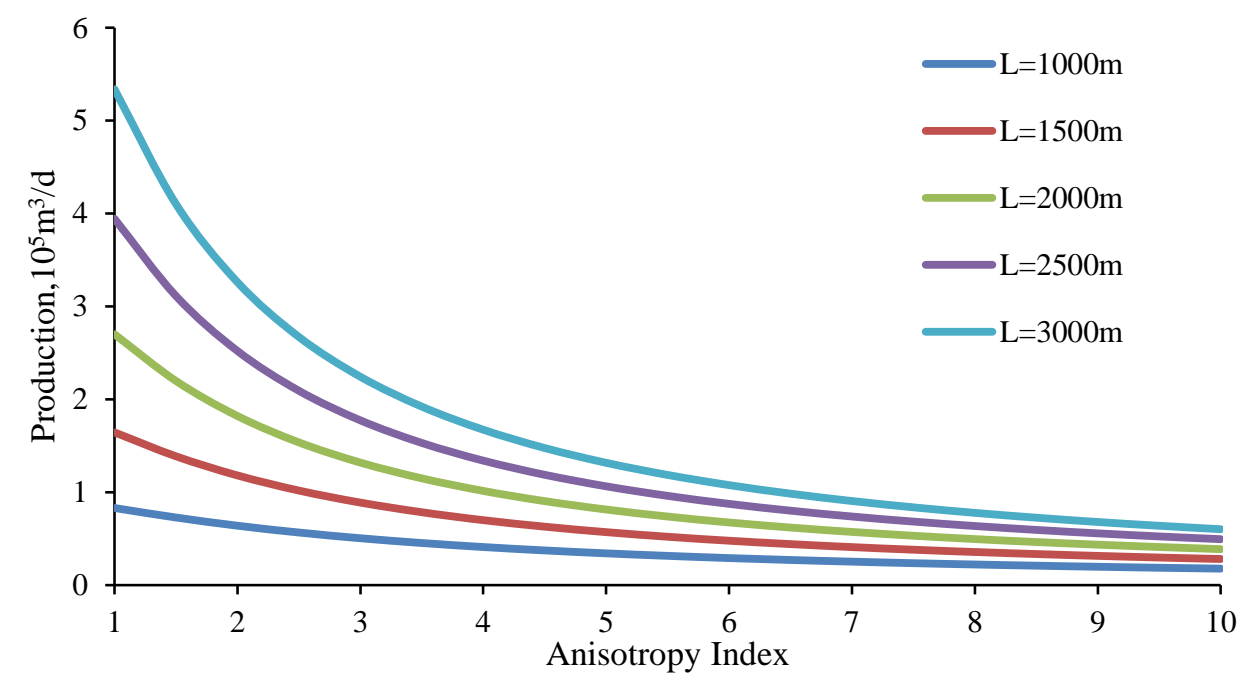

Figure 8-Effect of the anisotropy index on production at different horizontal well length

Eccentric distance. The influence of the eccentric distance $(\delta)$ on production was investigated versus various horizontal well length. The anisotropy index $\left(I_{a n i}\right)$, was set to be 1 . Figure 9 shows that the larger the eccentricity distance, the smaller the production, and the degree of influence gradually decreases. The longer the horizontal well is, the more impact of eccentricity distance on productivity.

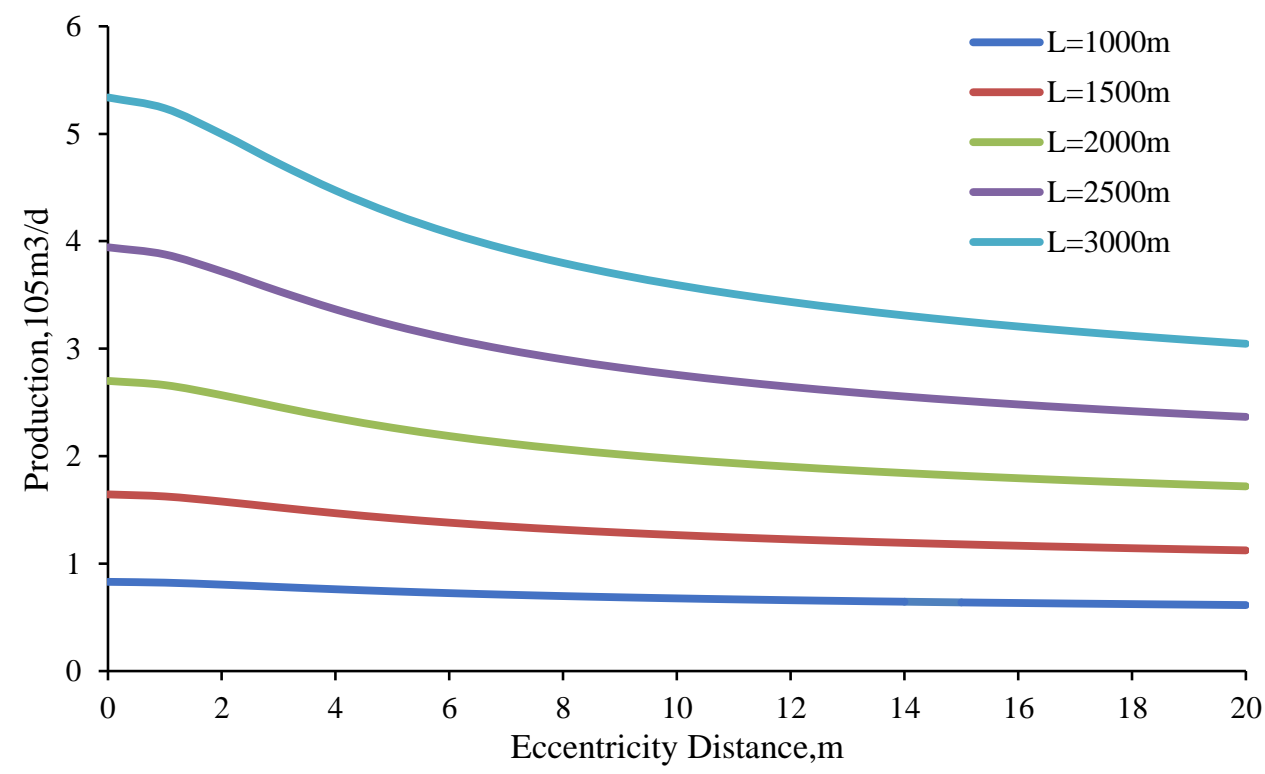

Figure 9-Effect of the eccentricity distance on production at different horizontal well length

Formation damage. Figure 10 depicts the influence of formation damage. It indicates that the longer the horizontal well is, the more impact of skin factor $(S)$ on horizontal well production. 


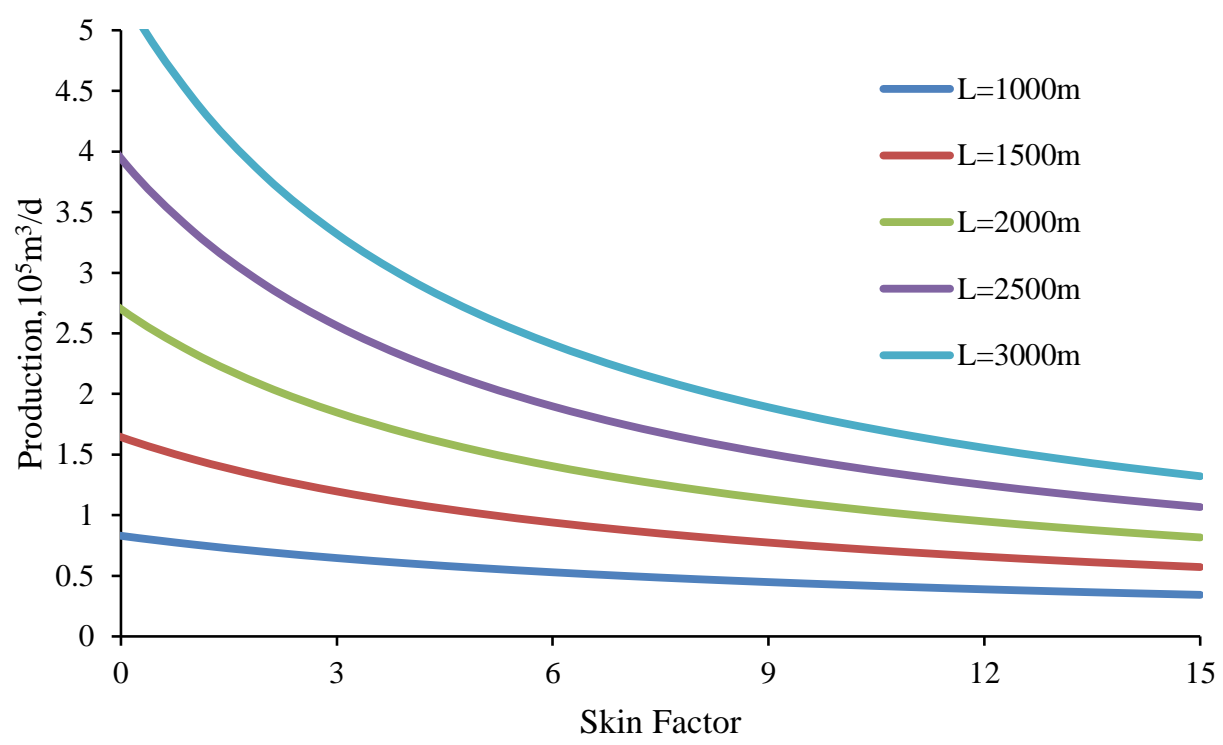

Figure 10 — Effect of the formation damage on production at different horizontal well length

\section{Conclusions}

A new horizontal well productivity evaluation model based on stress sensitive permeability in an elliptical reservoir is established. And the proposed productivity model was extended to consider the effects of reservoir heterogeneity, eccentricity, and formation damage on horizontal well productivity. The following conclusions can be drawn:

1. The thinner the formation is, the greater the impact of the horizontal well lengths on production. Thus, horizontal well is suggested in a thin formation.

2. The more stress sensitive the formation is, the lower the production. As the horizontal well length is longer, the impact of stress sensitivity on the production becomes more significant.

3. The closer reservoir shape is to the circle, the lower the production of horizontal well. Thus, horizontal well would be a better well type option for elliptical reservoirs.

4. The longer the horizontal well is, the more impact of heterogeneity, eccentricity distance, as well as skin factor on productivity.

\section{Acknowledge}

Acknowledgment to the following projects for supporting the research work of this paper: Scientific Research Project of Key Laboratory of Education Department in Shaanxi Province: "Research on well test evaluation technology of unconventional horizontal well exploitation" (13JS090)

\section{Conflicts of Interest}

The author(s) declare that they have no conflicting interests.

\section{Nomenclature}

$a=$ the semi-major axis of the elliptical drainage area formed in the horizontal wells, $m$

$b=$ the short half axis of the ellipsoidal drain region formed in the horizontal wells, $m$

$B=$ formation volume factor

$h=$ reservoir thickness, $\mathrm{m}$ 


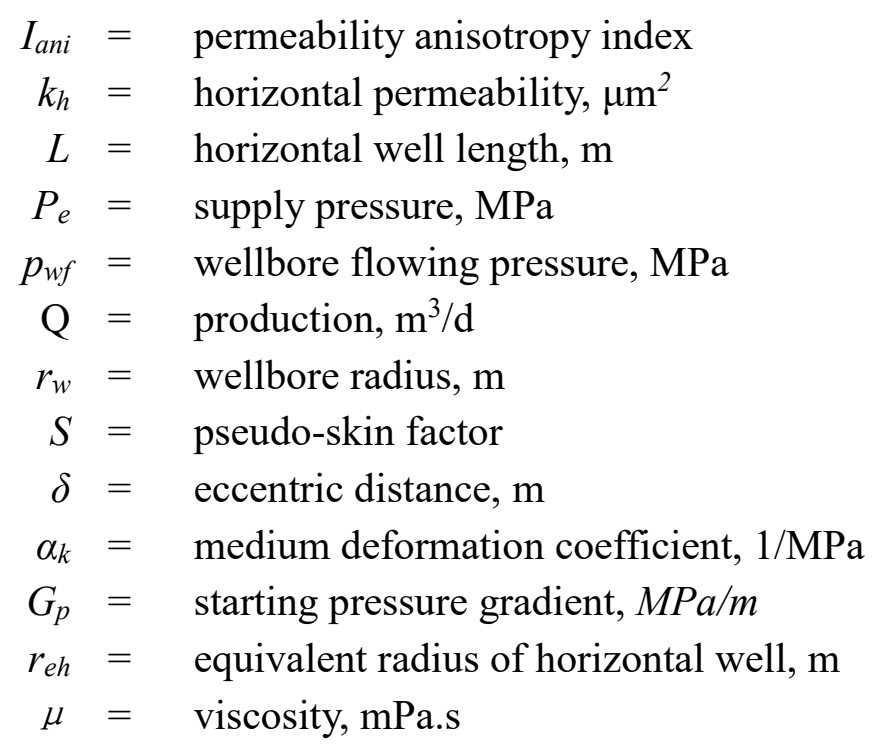

\section{Reference}

Anklam, E.G. and Wiggins, M.L. 2005. Horizontal Well Productivity and Wellbore Pressure Behavior Incorporating Wellbore Hydraulics. Paper presented at SPE Production Operations Symposium, Oklahoma City, Oklahoma, 1619 April. SPE-94316-MS.

Babu, D.K. and Odeh, A.S. 1988. Productivity of a Horizontal Well. Paper presented at SPE Annual Technical Conference and Exhibition, Houston, Texas, 2-5 October. SPE-18334-MS.

Billiter, T., Lee, J., and Chase, R. 2001. Dimensionless Inflow-Performance-Relationship Curve for Unfractured Horizontal Gas Wells. Paper presented at SPE Eastern Regional Meeting, Canton, Ohio, 17-19 October. SPE72361-MS.

Chen, M., Pu, C., and Zhao, J. 2006. Calculation and Analysis of the True Productivity of a Well of Low Permeable Reservoirs of Deformation Medium. Journal of Xi' an Shiyou University (Natural Science Edition) 21(2):18-23.

Chen, M., Zhang, M., and Pu, C. 2007. Productivity of Horizontal Well in Deformation Medium of Low-Permeability Reservoirs. Acta Petrolei Sinica 28(1): 107-110.

Chen, Y. and Zou, C. 2009. Derivation, Comparison and Application of Horizontal Well Production Formula Considering Anisotropy and Eccentricity Effects. Xinjiang Petroleum Geology 30(4):486-489.

Chen, Y., Hao, M., Sun, B., et al. 2012. Comparative Study of the Production Rate Formulas for Horizontal Wells. Xinjiang Petroleum Geology 33(5):566-569.

Chen, X., Mu, L., and Wu, Z. 2019. Calculating Method of the Productivity for the Trans-Layer Fractured Horizontal Wells. Petroleum Geology \& Oilfield Development in Daqing 38(3):65-72.

Guo, B., Ling, K., and Ghalambor, A. 2006. A Rigorous Composite-IPR Model for Multilateral Wells. Paper Presented at SPE Annual Technical Conference and Exhibition, San Antonio, Texas, USA, 24-27 September. SPE100923-MS.

Giger, F., Reiss, L.H., and Jourdan, A. 1984. The Reservoir Engineering Aspects of Horizontal Drilling. Paper presented at SPE Annual Technical Conference and Exhibition, Houston, Texas, 16-19 September. SPE-13024MS.

Hagoort, J. 2011. Semisteady-State Productivity of a Well in a Rectangular Reservoir Producing at Constant Rate or Constant Pressure. SPE Reservoir Evaluation \& Engineering 14(6):677-686. SPE-149807-PA.

Joshi, S. D. 1988. Augmentation of Well Productivity with Slant and Horizontal. Journal of Petroleum Technology 40(6):729-739. SPE-15375-PA.

Joshi, S. D. 1988. Production Forecasting Methods for Horizontal Wells. Paper presented at International Meeting on Petroleum Engineering, Tianjin, China, 1-4 November. SPE-17580-MS. 
Kewen, L. and Chen, Z. 2012. Computation of Productivity Index with Capillary Pressure Included and Its Application in Interpreting Production Data from Low-Permeability Oil Reservoirs. SPE Journal 17(4):10411046. SPE-146648-PA.

Larsen, L. 1996. Productivity Computations for Multilateral, Branched and Other Generalized and Extended Well Concepts. Paper presented at SPE Annual Technical Conference and Exhibition, Denver, Colorado, 6-9 October. SPE-36754-MS.

Lu, J. and Tiab, D. 2007. A Simple Productivity Equation for a Horizontal Well in Pseudo-Steady State in A Closed Anisotropic Box Shaped Reservoir. Paper presented at Production and Operations Symposium, Oklahoma City, Oklahoma, U.S.A., 31 March-3 April. SPE-106970-MS.

Peaceman, D.W. 1990. Discussion of Productivity of a Horizontal Well. SPE Reservoir Engineering 5(2): 252-253. SPE-31025-PA.

Penmatcha, V.R. and Khalid, A. 1999. Effects of Pressure Drop in Horizontal Wells and Optimum Well Length. Paper presented at SPE Production Operations Symposium, Oklahoma City, Oklahoma, 9-11 March. SPE-37494-MS.

Renard, G. and Dupuy, J. M. 1991. Formation Damage Effects On Horizontal-Well Flow Efficiency. Journal of Petroleum Technology 43(7):786-869. SPE-19414-PA.

Salam, A. 2019. Revisiting Current Techniques for Analyzing Reservoir Performance: A New Approach for Horizontal-Well Pseudosteady-State Productivity Index. SPE Journal 24(1):71-91. SPE-191139-PA.

Sun, R., Zhang, Y., and Zhang, X. 2019. Productivity Prediction Model and Parameter Analysis for Fractured Horizontal Wells in Double Medium Tight Oil Reservoirs. Science Technology and Engineering 19(10):89-96.

Tabatabaei, M., Mack, D.J., and Daniels, N.R. 2009. Evaluating the Performance of Hydraulically Fractured Horizontal Wells in the Bakken Shale Play. Paper presented at SPE Rocky Mountain Petroleum Technology Conference, Denver, Colorado, 14-16 April. SPE-122570-MS.

Yildiz, T. 2003. Mutilateral Pressure-Transient Response. SPE Journal 8(1):234-245. SPE-83631-PA.

Yuan, B., Su, Y., and Rouzbeh, G.M. 2015. A New Analytical Multi-Linear Solution for Gas Flow toward Fractured Horizontal Wells with Different Fracture Intensity. Journal of Natural Gas Science and Engineering 23(2015):227-238.

Yuan, Y., Zhang, L., Wang, J., et al. 2009. A Binomial Deliverability Equation for Horizontal Gas Wells in Formations with Nonlinear Seepage Flow Features. Oil \&Gas Geology 30(1):122-126.

Zhu, D., Hill, A. D., and Landtum, W. R. 2002. Evaluation of Crossflow Effects in Multilateral Well. Paper presented at SPE/DOE Improved Oil Recovery Symposium, Tulsa, Oklahoma, 13-17 April. SPE-75250-MS.

Weidong Tian, is a master candidate in Petroleum Engineering department at Xi'an Shiyou University. He has focused his research in areas involving reservoir simulation, well testing, and production analysis. Tian holds an BS degree in Petroleum Engineering from Xi'an Shiyou University.

Zhenzhen Dong is a Professor in the Petroleum Engineering Department at Xi'an Shiyou University. She worked as a reservoir engineer with Schlumberger from 2012 to 2016 . Her research interests include unconventional resources/reserves estimates, reservoir simulation, well testing, and production analysis. Dong holds a bachelor's degree in mathematics from Northeast Petroleum University, China; a master's degree in petroleum engineering from Research Institute of Petroleum Exploration and Development, China; and a PhD degree in petroleum engineering from Texas A\&M University.

Jiaen Lin is a Professor of Petroleum Engineering Department at Xi'an Shiyou University, where he has worked as faculty for the last 30 years. He focuses his research in well testing and production analysis. 